\title{
Role of the triplet state in the green emission peak of polyfluorene films: A time evolution study
}

\author{
S. Mukhopadhyay, ${ }^{1, \text { a) }}$ S. Ramasesha, ${ }^{1, b)}$ and S. Guha ${ }^{2, c)}$ \\ ${ }^{1}$ Solid State and Structural Chemistry Unit, Indian Institute of Science, Bangalore 560012, India \\ ${ }^{2}$ Department of Physics and Astronomy, University of Missouri, Columbia, Missouri 65211, USA
}

(Received 5 November 2009; accepted 4 January 2010; published online 25 January 2010)

\begin{abstract}
The blue emission of ethyl-hexyl substituted polyfluorene (PF2/6) films is accompanied by a low energy green emission peak around $500 \mathrm{~nm}$ in inert atmosphere. The intensity of this $500 \mathrm{~nm}$ peak is large in electroluminescence (EL) compared to photoluminescence (PL) measurements. Furthermore, the green emission intensity reduces dramatically in the presence of molecular oxygen. To understand this, we have modeled various nonradiative processes by time dependent quantum many body methods. These are (i) intersystem crossing to study conversion of excited singlets to triplets leading to a phosphorescence emission, (ii) electron-hole recombination (e-hR) process in the presence of a paramagnetic impurity to follow the yield of triplets in a polyene system doped with paramagnetic metal atom, and (iii) quenching of excited triplet states in the presence of oxygen molecules to understand the low intensity of EL emission in ambient atmosphere, when compared with that in nitrogen atmosphere. We have employed the Pariser-Parr-Pople Hamiltonian to model the molecules and have invoked electron-electron repulsions beyond zero differential approximation while treating interactions between the organic molecule and the rest of the system. Our time evolution methods show that there is a large cross section for triplet formation in the e-hR process in the presence of paramagnetic impurity with degenerate orbitals. The triplet yield through e-hR process far exceeds that in the intersystem crossing pathway, clearly pointing to the large intensity of the $500 \mathrm{~nm}$ peak in EL compared to PL measurements. We have also modeled the triplet quenching process by a paramagnetic oxygen molecule which shows a sizable quenching cross section especially for systems with large sizes. These studies show that the most probable origin of the experimentally observed low energy EL emission is the triplets. (c) 2010 American Institute of Physics. [doi:10.1063/1.3298371]
\end{abstract}

\section{INTRODUCTION}

Pristine sample of polyfluorene type materials are widely used in polymer based light-emitting devices due to their emission in the blue region of the electromagnetic spectrum $(\sim 3.0 \mathrm{eV}){ }^{1,2}$ As a result of their large energy gaps, they can also be used as host materials for internal color conversion and their liquid crystalline behavior allows realization of polarized light emission. ${ }^{3,4}$ Conjugated molecular solids are van der Waals solids and hence their photophysics is dominated by Frenkel excitons. The present study is motivated by strikingly different electroluminescence (EL) with respect to photoluminescence (PL) (under ambient as well as $\mathrm{N}_{2}$ atmosphere) of polyfluorene films as reported by various groups. ${ }^{5,6}$ Backed by few experimental evidences, such a difference is assigned to defects in the chemical structure (ketodefect) manifested as fluorenones. ${ }^{7,8}$ On the contrary, a large body of experimental results point to an intrinsic phenomenon rather than to a trap created by chemical defects. ${ }^{9}$ One of the most striking features of polyfluorene (PF) films is the presence of a triplet state around 2.2-2.3 eV (Ref. 10) which

\footnotetext{
${ }^{a)}$ Electronic mail: sukrit@sscu.iisc.ernet.in.

${ }^{b)}$ Electronic mail: ramasesh@sscu.iisc.ernet.in.

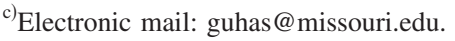

is exploited for preparing highly efficient electrophosphorescent devices. Recent EL studies on PF films show emission at $2.4 \mathrm{eV}$ in nitrogen atmosphere which gets reduced under ambient conditions. ${ }^{11}$ While conventional wisdom associates the low energy EL peak to keto-defects, the fact that it quenches in an oxygen atmosphere where a keto-defect should be dominant, makes it an unlikely origin of the $2.4 \mathrm{eV}$ EL peak. Figure 1 highlights the differences in PL and EL from an ethyl-hexyl substituted polyfluorene (PF2/6) lightemitting diode which was fabricated without exposing the polymer layer to atmospheric oxygen. Details of our experimental results are found in Ref. 11. In this paper, we attribute the origin of the $2.4 \mathrm{eV}$ EL peak in nitrogen atmosphere to the emission from the triplet state. Absence of this EL peak in oxygen atmosphere is attributed to quenching of the triplets by paramagnetic oxygen molecules. We substantiate this by theoretical studies of different photophysical processes. We have used a time dependent quantum many-body method which was described briefly in Ref. 11. In this article, we present a detailed study of various time dependent processes and also simulate the effect of keto-defects on these processes. We also model the effect of doped metal atoms on the EL emission to understand the role of elements such as $\mathrm{Cu}$ and $\mathrm{Pd}$ on the electronic processes occurring in PF films. 


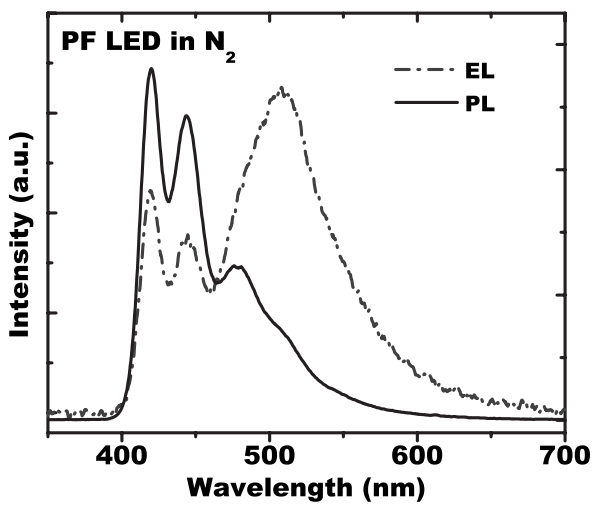

FIG. 1. EL and PL spectra from PF2/6 LED for device fabricated in $\mathrm{N}_{2}$ atmosphere.

Theoretical calculations shown below are directed to substantiate the involvement of the triplet state in the EL spectrum.

\section{MODEL HAMILTONIAN}

Our theoretical model system consists of a conjugated finite polyene chain and a paramagnetic impurity (metal atom/oxygen molecule). We wish to model the process of (i) intersystem crossing (ISC) of an excited singlet $\left(S_{n}\right)$ of a polymer/molecule to a triplet state by interaction with a paramagnetic atom or an organic radical (ii) electron-hole recombination process (e-hR) in presence and absence of paramagnetic impurity leading to ground singlet state and the excited triplet or singlet states, and (iii) quenching of the triplet state $\left(T_{m}\right)$ of the polyene by the oxygen molecule or metal ion impurity. Although simple spin statistics place an upper limit on the quantum efficiencies for the formation of singlets in an e-hR process at $25 \%$, EL efficiencies in excess of $50 \%$ have been observed in highly ordered polymeric films. ${ }^{12}$ This has been studied by Hong et al. ${ }^{13}$ and Kobrak et al. ${ }^{14}$ by using models that incorporate electron phonon interactions. They argued that the injected electron and hole recombine preferentially to form a singlet exciton mainly because of its low binding energy and strong intrachain vibronic coupling. Karabunarliev et al. ${ }^{15}$ used similar models to discuss the effect of conjugation length and particle-hole symmetry on the polaron recombination process. In this study the authors incorporated electron phonon coupling and treated the electronic part at single configuration interaction level. Barford ${ }^{16}$ suggested that electron phonon coupling leads to preferential formation of interchain singlet excitons which later reconvert to intrachain singlet excitons. All these studies ignore explicit electron-electron correlations which can lead to wrong conclusions ${ }^{17}$ since conjugated molecules have been shown to be strongly interacting systems. Another approach to study the time dependent dynamics in conjugated polymers is the collective electronic oscillator model due to Mukamel and co-workers. ${ }^{18}$ This procedure assumes that the system can be described by the single Slater determinant constructed from an orthogonal one-electron basis and thus misses electron correlations. This study on the other hand incorporates electron correlations exactly within a zero differential overlap approximation for an isolated molecule and considers elec- tronic processes in the presence of doped species. Our model system for process (i) consists of a polymeric chain and a radical/metal atom whereas process (ii) is modeled using two polyene chains of opposite charges in presence and absence of a paramagnetic impurity and for process (iii) we consider a polymeric chain and an oxygen molecule in the triplet ground state or a metal ion impurity possessing an unpaired electron. The initial singlet, triplet, and the polaronic states of the polymer are the eigenstates within the Pariser-ParrPople (PPP) (Ref. 19) model with standard parameters and Ohno potentials ${ }^{20}$ given by

$$
V_{i j}=\left(\frac{U_{i}+U_{j}}{2}\right)\left[1+0.001206\left(U_{i}+U_{j}\right)^{2} r^{2}\right]^{-1 / 2}
$$

for long range Coulomb interactions. The radical/metal atom is described by a single nondegenerate or a doubly degenerate orbital occupied by an unpaired electron. In case of triplet quenching (TQ) the paramagnetic impurity of ISC is replaced by oxygen molecule described by the pair of doubly degenerate $\pi$ and $\pi^{*}$ orbitals with a total occupancy of six electrons in a triplet spin state. The model Hamiltonian for oxygen molecule spans the space of these four orbitals and the parameters are adjusted to obtain a triplet ground state separated from the lowest singlet excited state by an energy gap of $1.01 \mathrm{eV}$ which is close to the experimental value of $0.97 \mathrm{eV}^{21,22}$ The Hamiltonians for the polyenic moiety, oxygen molecule, and the paramagnetic impurity are given by

$$
\begin{gathered}
H_{\text {polymer }}^{\text {PPP }}=\sum_{i} \alpha_{i} \hat{E}_{i i}+\sum_{\langle i j\rangle} t_{i j} \hat{E}_{i j}+\frac{1}{2} \sum_{i} U_{i} \hat{n}_{i}\left(\hat{n}_{i}-1\right) \\
+\sum_{i>j} V_{i j}\left(\hat{n}_{i}-z_{i}\right)\left(\hat{n}_{j}-z_{j}\right) \\
H_{\mathrm{O}_{2}}=H_{\mathrm{PPP}}+\frac{1}{2} \sum_{i j k l}[i j \mid k l]\left(\hat{E}_{i j} \hat{E}_{k l}-\delta_{j k} \hat{E}_{i l}\right) \\
H_{P}=\alpha_{P} \hat{a}_{P, \sigma}^{+} \hat{a}_{P, \sigma}+\frac{U_{P}}{2} \hat{n}_{P}\left(\hat{n}_{P}-1\right)
\end{gathered}
$$

where $\hat{E}_{i j}=\Sigma_{\sigma} \hat{a}_{i, \sigma}^{+} \hat{a}_{j, \sigma}, \hat{a}_{i, \sigma}^{+}$and $\hat{a}_{j, \sigma}$ are the usual fermionic creation and annihilation operators, $\langle i j\rangle$ denotes the bonded pair, and $\hat{n}_{i}$ is the number operator for the site " $i$ ". $[i j \mid k l] \mathrm{s}$ are the two electron repulsion integrals in the charge cloud notation, $\alpha$ s are the site energies, $U$ s are the on-site repulsion integrals (Hubbard parameters) and $V_{i j} \mathrm{~s}$ are the long-range repulsion parameters obtained using Ohno parameterization. In ISC, e-hR and TQ processes, ${ }^{23}$ the total spin of the impurity and the polyene moiety are conserved, although the individual spins of the polyene and the impurity can undergo a change. This is very similar to the spin-orbital interaction process in which the z-components of spin and orbital angular momentum change even as the z-component of the total angular momentum is conserved. Such spin crossover processes are previously modeled in two different ways ${ }^{24,25}$ essentially arising from the overlap of polymeric atomic orbitals with that of impurity.

The charge transfer (CT) pathway is due to the oneelectron transfer integrals between impurity and polymer 


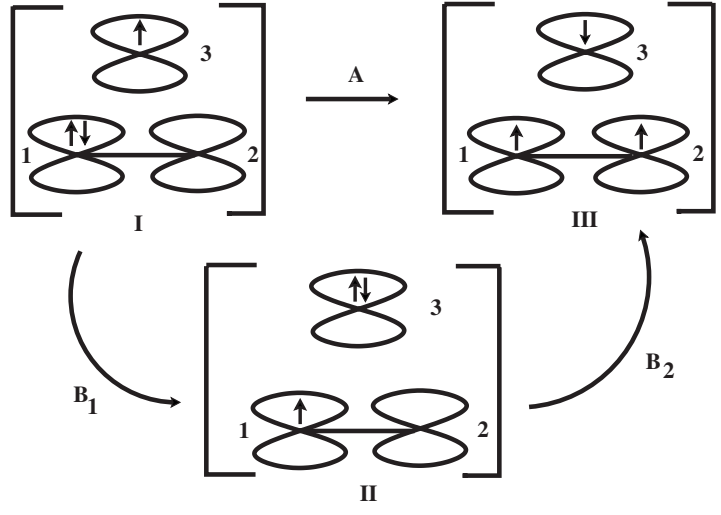

FIG. 2. The charge transfer $\left(\mathrm{B}_{1}\right.$ and $\left.\mathrm{B}_{2}\right)$ and the exchange (A) pathways for conversion of the singlet excited state of an ethylene molecule (represented by sites 1 and 2) to the triplet state by a paramagnetic impurity (site 3) are depicted here. The charge transfer pathway is mediated by the transfer integrals $\left(t_{31}^{\prime}\right.$ for $\mathrm{B}_{1}$ and $t_{23}^{\prime}$ for $\mathrm{B}_{2}$ ) and the exchange pathway is mediated through the two electron integral [13|23]. The corresponding operators for these two pathways are given in Eq. (3).

whereas the exchange pathway is due to the two electron integrals between the same entities. Both these pathways (Fig. 2) are incorporated through the interaction Hamiltonian $\left(H_{\text {int }}\right)$

$$
\begin{aligned}
H_{\mathrm{int}}= & \sum_{I j} t_{I j}^{\prime} \hat{E}_{I j}+\sum_{I p} t_{I p}^{\prime} \hat{E}_{I p}+\sum_{i p} t_{i p}^{\prime} \hat{E}_{i p} \\
& +\frac{1}{2} \sum_{I J k l}\left\{[I J \mid k l] \hat{E}_{I J} \hat{E}_{k l}+[I k \mid J l] \hat{E}_{I k} \hat{E}_{J l}\right\} \\
& +\frac{1}{2} \sum_{p p^{\prime} m n}\left\{\left[p p^{\prime} \mid m n\right] \hat{E}_{p p^{\prime}} \hat{E}_{m n}+\left[p m \mid p^{\prime} n\right] \hat{E}_{p m} \hat{E}_{p^{\prime} n}\right\}
\end{aligned}
$$

where $t_{I j}^{\prime}, t_{I p}^{\prime}$, and $t_{i p}^{\prime}$ are the one-electron transfer integral giving rise to the CT process and $[I J \mid k l] \mathrm{s}$ are the two electron exchange integrals in the charge cloud notation. Lower case indices correspond to orbitals on the first chain, upper case $I$ and $J$ correspond to orbitals on the second chain, $p$ and $p^{\prime}$ correspond to orbitals on PM, and indices $m$ and $n$ in the last summation run over orbitals on both chains. In case of $\mathrm{e}-\mathrm{hR}$, terms involving the paramagnetic species occur only when the system is studied in presence of a paramagnetic moiety. In case of ISC, the interaction Hamiltonian [Eq. (3)] will contain only those terms which involve lower case indices, because our model consist of only one polyene chain along with the PM.

\section{CHOICE OF PARAMETERS AND MOLECULAR GEOMETRIES}

This section mainly focuses on the geometry of the total system and the parameters used for one and two electron integrals. The geometry of the polyene chain (Fig. 3) used in our calculations have alternate long and short bonds which is also reflected in the transfer integrals according to the equation

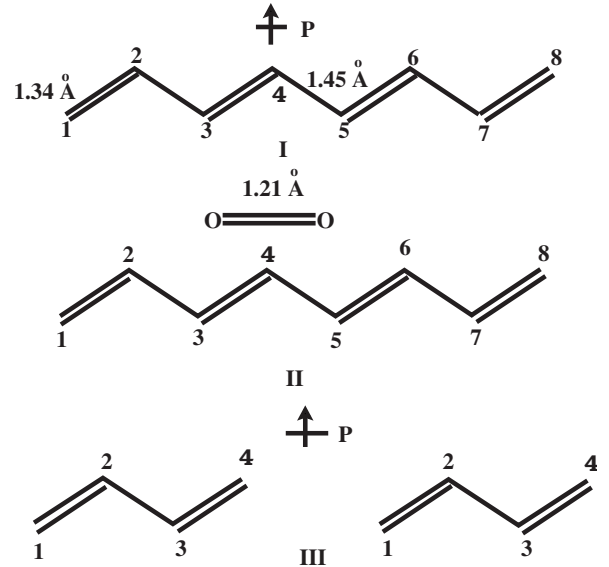

FIG. 3. The geometries of the collision complexes used in our time dependent calculation to model (i) ISC, (ii) triplet annihilation in presence of oxygen (TQ), and (iii) e-hR in presence of paramagnetic impurity.

$$
t=t_{0}(1 \pm \delta),
$$

where $t_{0}$ is the transfer integral for a benzenoid $\mathrm{C}-\mathrm{C}$ bond and $\delta$ is the bond alternation parameter. The parameters used are $t_{0}=-2.4 \mathrm{eV}, \delta=0.07$, and Hubbard $U_{C}=11.26 \mathrm{eV}$ with Ohno scheme for intersite interactions given by $V_{i j}$ according to Eq. (1) by substituting $U_{i}=U_{j}=U_{C}$. The long axis of the polyene is placed along the $x$-axis. The paramagnetic metal sites are placed (Fig. 3) about $3.8 \AA$ away from the center of the polymeric chain along the $y$-direction. The paramagnetic metal site is modeled as consisting of one orbital or two degenerate orbitals occupied by a single electron. The difference in bond-lengths of the metal-carbon (M-C) bond and carbon-carbon $(\mathrm{C}-\mathrm{C})$ bond leads us to conclude that the metal $d$-orbital has larger spatial extent than the carbon $2 p_{z}$ orbitals by about $1 \AA^{26}{ }^{26}$ Considering this fact the oneelectron transfer integrals $\left(t_{i j}\right)$ between metal site and polymer sites are calculated by an empirical formulae

$$
t_{i j}=\exp \left(\frac{d_{i j}-2.34}{0.529}\right) 2.57,
$$

where $d_{i j}$ is the distance between two sites in $\AA$ and the transfer integrals are in $\mathrm{eV}$. The two electron integrals, beyond PPP interactions, that are included are the bond-charge repulsion integrals $([i i \mid k l])$ and the bond-bond repulsion integrals $([i j \mid k l])$ where a chemical bond is assumed to exist between the pair of sites $i$ and $j$ as well as $k$ and $l$. The parameterization of these integrals are given by

$$
\begin{aligned}
& {[i i \mid k l]=\exp \left(-\frac{d_{i, k l}}{0.529}\right)[i i \mid k k],} \\
& {[i j \mid k l]=\exp \left(-\frac{d_{i j, k l}}{0.529}\right)[i i \mid k k],}
\end{aligned}
$$

where $d_{i, k l}$ is the distance between the $i$ th site and the midpoint of " $k l$ " bond and $d_{i j, k l}$ is the distance between midpoints of " $i j$ " and " $k l$ " bonds. [ $i i \mid k k]$ is the PPP interaction between the two sites $i$ and $k$ in the Ohno scheme according to Eq. (1). Under such assumptions the largest $[i i \mid k l]$ integral assumes a value of $0.50 \mathrm{eV}$ and the largest value for $[i j \mid k l]$ 
TABLE I. The Oxygen parameters beyond PPP Hamiltonian (all parameters in $\mathrm{eV}$ ). See text for symbols.

\begin{tabular}{lcccccc}
\hline \hline$\alpha_{0}$ & $U_{p}$ & $U_{p p}$ & $J_{p p}$ & $W_{p p}$ & {$\left[p_{1} p_{1} \mid p_{1} p_{3}\right]$} & {$\left[p_{1} p_{3} \mid p_{1} p_{3}\right]$} \\
\hline-3.00 & 13.08 & 10.00 & 5.00 & 2.50 & 0.37 & 0.15 \\
\hline \hline
\end{tabular}

integral is about an order of magnitude less than this value. We have varied the site energies of the metal site from 2.0 to $5.0 \mathrm{eV}$ because of ultraviolet photoelectron spectroscopy (UVPES) (Ref, 27) data which suggests that the $3 d / 4 d$ orbitals of metals such as $\mathrm{Cu}, \mathrm{Re}, \mathrm{Ir}$, and $\mathrm{Pt}$ are placed roughly 3.0-5.0 eV above the carbon $2 p$ orbital. The Hubbard $U$ of the metal orbital is fixed at $8.0 \mathrm{eV}$ which is smaller than $U_{C}$ and reflects the larger spatial extent of the $d$-orbitals. We have also considered the case in which one unpaired electron is occupied in two degenerate orbitals on the paramagnetic moiety for a few of the above mentioned parameter sets to mimic a $d$-manifold. The repulsion integral between a pair of electrons in the doubly degenerate paramagnetic metal orbitals is taken to be $6.0 \mathrm{eV}$. For parameters on the oxygen molecule, we consider two orbitals $p_{1}$ and $p_{2}$ on one oxygen atom and two more orbitals $p_{3}$ and $p_{4}$ on the other. The integrals that we consider in our study are $U_{p}=\left[p_{1} p_{1} \mid p_{1} p_{1}\right], \quad U_{p p}=\left[p_{1} p_{1} \mid p_{2} p_{2}\right], \quad J_{p p}=\left[p_{1} p_{1} \mid p_{2} p_{1}\right]$, and $W_{p p}=\left[p_{1} p_{2} \mid p_{1} p_{2}\right]$ which are localized on either of the oxygen atoms whereas integrals such as $\left[p_{1} p_{1} \mid p_{1} p_{3}\right]$ and $\left[p_{1} p_{3} \mid p_{1} p_{3}\right]$ are delocalized between the two oxygen atoms. The values of these integrals assumed in our study are listed in Table I. We have checked that these parameters yield a triplet ground state for the molecule with a separation of $1.01 \mathrm{eV}$ from the lowest singlet state. The experimental value of this gap is $0.97 \mathrm{eV}^{28}$

We have studied the TQ process for different distances between the oxygen molecule and the polyene molecule; the relative geometry is shown in (II) of Fig. 3. The transfer and exchange integrals are calculated in the same manner as described for paramagnetic metal impurity.

\section{WAVE PACKET DYNAMICS}

We study the ISC, e-hR, and TQ processes by starting with an initial wave-packet corresponding to the subsystems which are noninteracting and evolving them with time under the influence of the full Hamiltonian $\left(H_{\mathrm{tot}}\right)$. Thus, given an initial state $\Psi(0)$, we solve the time dependent Schrödinger equation

$$
i \hbar \frac{\partial \Psi(t)}{\partial t}=H_{\text {total }} \Psi(t) .
$$

The formal solution of the above equation [Eq. (7)] is expressed by the time evolution operator which is represented by an exponential function of the Hamiltonian matrix

$$
\psi(t)=\exp \left(\frac{-i H_{\text {tot }} t}{\hbar}\right) \psi(0) .
$$

To evaluate such expressions exactly, we must know the total eigenspectrum of the Hamiltonian; a daunting task for dimensions larger than a few thousands since we evolve the full N-electron wave-function at every time step. Thus, obtaining the full eigenvalue spectrum even for the interacting many-body subsystems becomes quite prohibitive. Hence we use the Schrödinger representation where the Hamiltonian of the full system is given by

$$
H_{\text {tot }}=H_{\text {polymer }}^{\mathrm{PPP}}+H_{P_{/} \mathrm{O}_{2}}+H_{\mathrm{int}} .
$$

We use a discretized time evolution scheme to solve Eq. (8). In the discretized time evolution procedure, the wavefunction at time " $t+\Delta t$ " is obtained from that at time " $t$ " by approximating the time evolution operator $\exp (-i H t / \hbar)$.

Various schemes have been proposed to approximate this exponential function; the most widely used is the CrankNicholson (CN) scheme. In this scheme the exponential function is approximated by Cayley transform

$$
|\Psi, t+\Delta t\rangle=\frac{1-i H \Delta t / 2 \hbar}{1+i H \Delta t / 2 \hbar}|\Psi, t\rangle+O\left((H \Delta t)^{3}\right) .
$$

This scheme is unitary, unconditionally stable and accurate up to $O(H \Delta t)^{2}$. Although the inversion of the large matrix $(1+i H \Delta t / 2 \hbar)$ can be done using the small matrix technique, this implicit method is prohibitive because of large CPU time requirement. Another scheme which is straight forward to implement is the multi-step differencing scheme (MSD) ${ }^{29}$ It is symmetric in time and is shown to be conditionally stable. Further the scheme is accurate up to $O(H \Delta t)^{5}$. Here we have used the fourth order MSD scheme (MSD4) (Ref. 30) given by the expression

$$
\begin{aligned}
|\Psi, t+4 \Delta t\rangle= & |\Psi, t\rangle-4 i H \Delta t\left[-\frac{1}{3}|\Psi, t+2 \Delta t\rangle\right. \\
& \left.+\frac{2}{3}(|\Psi, t+3 \Delta t\rangle+|\Psi, t+\Delta t\rangle)\right] .
\end{aligned}
$$

Initially $|\Psi, t+\Delta t\rangle,|\Psi, t+2 \Delta t\rangle,|\Psi, t+3 \Delta t\rangle$ are obtained by the $\mathrm{CN}$ technique and the MSD4 scheme is used to obtain the wave-function at later time steps. The stability criterion for this scheme is given by

$$
\alpha=\left|E_{m}\right| \Delta t<0.4,
$$

where $E_{m}$ is the maximum eigenvalue for the given Hamiltonian.

\section{INITIAL STATE CONSTRUCTION AND COMPUTATION OF TIME DEPENDENT YIELDS}

In the wave-packet dynamics, the first task is to construct an appropriate wave-packet from the eigenstates of the subsystem and propagate the wave-packet employing the full Hamiltonian. Depending upon the process we wish to study, the initial states would be different. In this section we describe the construction of the initial state for the ISC, e-hR and TQ processes.

\section{A. Wave-packet dynamics for ISC study}

We construct the initial state for the intersystem crossing process as a direct product of the singlet excited state of the molecule $\left(S_{n}\right)$ and the ground state of the paramagnetic atom or molecule $\left(\left|S_{n}\right\rangle \otimes|P\rangle\right)$ 


$$
\Psi_{\text {ISC }}(0)=|0,0\rangle_{n} \otimes\left|\frac{1}{2}, \frac{1}{2}\right\rangle,
$$

where $|0,0\rangle_{n}$ is the $n$th excited singlet state of the polyene expressed as a linear combination of the singlet valencebond (VB) (Ref. 31) functions which span the singlet space of the Hamiltonian and $|1 / 2,1 / 2\rangle$ is the state in which an unpaired electron is occupying an atomic orbital of the paramagnetic moiety. $\Psi_{\text {ISC }}(0)$ is evolved using the total Hamiltonian of the system given by

$$
H_{\text {tot }}=H_{\text {polymer }}^{\mathrm{PPP}}+H_{P}+H_{\mathrm{int}} .
$$

The wave-packet $\Psi_{\text {ISC }}(0)$ is time evolved using the time dependent Schrödinger equation according to the standard techniques described above. Recognizing the fact that the full system possesses a total spin $S=1 / 2$ and total z-component of spin $M_{s}=1 / 2$ and that the total Hamiltonian is nonrelativistic (does not contain spin-orbit interactions) the total spin value is conserved. However, the above total $S$ and $M_{s}$ can be arrived at, from $\left|T_{m}\right\rangle=|1,0\rangle$ or $|1,1\rangle$ corresponding to the impurity states $\left|\frac{1}{2}, \frac{1}{2}\right\rangle$ and $\left|\frac{1}{2},-\frac{1}{2}\right\rangle$, respectively. Therefore to obtain the yield of the triplet state in ISC process, the evolved state $\Psi_{\text {ISC }}(t)$ is projected on to the direct product of the desired triplet eigenstates of the molecular Hamiltonian and the impurity state $\left|\Psi_{T_{m}, P}\right\rangle$ given by

$$
\begin{aligned}
\left|\Psi_{T_{m}, P}\right\rangle= & \sqrt{\frac{1}{3}}|1,0\rangle_{m} \otimes\left|\frac{1}{2}, \frac{1}{2}\right\rangle \\
& -\sqrt{\frac{2}{3}}|1,1\rangle_{m} \otimes\left|\frac{1}{2},-\frac{1}{2}\right\rangle,
\end{aligned}
$$

with $|1,0\rangle_{m}$ representing the $m$ th $S=1, M_{s}=0$ eigenstate of the polymer Hamiltonian. The coefficients in Eq. (15) are the Clebsch-Gordon coefficients. Here, we have used the fact that total $S=1 / 2$ state can be obtained from the two subsystem with spins of $S_{1}=0$ and $S_{2}=1 / 2$ or $S_{1}=1$ and $S_{2}=-1 / 2$. The $|1,1\rangle_{m}$ state can be obtained from the $|1,0\rangle_{m}$ state by operating with the spin angular momentum ladder operator $S^{+}=\sum_{i} s_{i}^{+}$where " $i$ " runs over all $\pi$-orbitals used for describing the conjugated molecule. The probability at time " $t$ " that the system is in the triplet state $\left|T_{m}\right\rangle$ is given by

$$
P_{T_{m}}^{\mathrm{ISC}}(t)=\left|C_{T_{m}}(t)\right|^{2} ; C_{T_{m}}(t)=\left\langle\Psi_{\mathrm{ISC}}(t) \mid \Psi_{T_{m}, P}\right\rangle .
$$

The time evolution is followed as a function of time up to $t_{\max }=66 \mathrm{fs}$ for several low-lying triplet states. In our model, the nearest neighbor charge transfer integral due to the paramagnetic impurity is of the order of $0.5 \mathrm{eV}$, which implies a time scale of about 1.3 fs. Therefore, evolving the wavepacket for the duration of $66 \mathrm{fs}$ is expected to be sufficient to capture the effect of the paramagnetic impurity. The time integrated yield is given by

$$
I_{T}=\sum_{m} \int_{0}^{t_{\max }} P_{T_{m}}^{\mathrm{ISC}}(t) d t
$$

$I_{T}$ is the total yield of the triplet states within time $t_{\max }$. It is also a measure of the yield of the lowest triplet state since this state can be obtained from any of the higher lying triplet states via internal conversion in the triplet manifold as laid out by Kasha's rule. ${ }^{32}$

While the triplet states are generated by ISC from the singlet excited states, they are also annihilated (TA) by the interaction with the paramagnetic impurity. This is also an ISC process where the initial state of the polyene is the lowest triplet state and the final state is the ground singlet state. The time integrated yield for TA process in presence of paramagnetic metal impurity is given by

$$
\begin{aligned}
& I_{\mathrm{TA}}=\int_{0}^{t_{\max }} P_{S_{0}}^{\mathrm{TA}}(t) d t \\
& \left.\left.P_{S_{0}}^{\mathrm{TA}}(t)=\left|\left\langle\psi_{T_{1}, P}|| 0,0\right\rangle_{1} \otimes\right| \frac{1}{2}, \frac{1}{2}\right\rangle\right\rangle\left.\right|^{2} .
\end{aligned}
$$

Since the phosphorescence is a measure of radiative decay from the lowest triplet state, its intensity depends on the difference in the rates of the above two ISC processes. Therefore $\left(I_{T_{m}}-I_{\mathrm{TA}}\right)$ is a measure of the phosphorescence intensity.

\section{B. Wave-packet dynamics for charge recombination process}

To model the charge recombination phenomenon which is dominant in the electroluminescence process, we construct the initial state as a direct product of the ground states of positive and negative polarons along with a paramagnetic impurity. The initial state for such a process is given by

$$
\begin{aligned}
\psi_{\mathrm{e}-\mathrm{hR}}^{11 / 2,1 / 2\rangle}(0)= & \frac{1}{\sqrt{6}}\left|\frac{1}{2},-\frac{1}{2}\right\rangle_{M^{+}} \otimes\left|\frac{1}{2}, \frac{1}{2}\right\rangle_{M^{-}} \otimes\left|\frac{1}{2}, \frac{1}{2}\right\rangle_{P} \\
& +\frac{1}{\sqrt{6}}\left|\frac{1}{2}, \frac{1}{2}\right\rangle_{M^{+}} \otimes\left|\frac{1}{2},-\frac{1}{2}\right\rangle_{M^{-}} \otimes\left|\frac{1}{2}, \frac{1}{2}\right\rangle_{P} \\
& -\frac{2}{\sqrt{6}}\left|\frac{1}{2}, \frac{1}{2}\right\rangle_{M^{+}} \otimes\left|\frac{1}{2}, \frac{1}{2}\right\rangle_{M^{-}} \otimes\left|\frac{1}{2},-\frac{1}{2}\right\rangle_{P},
\end{aligned}
$$

where the subscript $M^{+}$and $M^{-}$stand for the lowest energy positive and negative polaronic states of the molecule whereas $P$ denotes the state of the paramagnetic impurity. The initial state $\psi_{\mathrm{e}-\mathrm{hR}}(0)$ possesses a total spin of $1 / 2$ and its corresponding z-component is also $1 / 2 .|1 / 2,1 / 2\rangle_{M^{+}}$ $\otimes|1 / 2,1 / 2\rangle_{M^{-}}$is the direct product of the ground state of the positively and negatively charged polyene, each state being expressed as a linear combination of the VB functions which spans the doublet space of the Hamiltonian. The $\mid 1 / 2$, $-1 / 2\rangle_{M^{+}}$and $|1 / 2,-1 / 2\rangle_{M^{-}}$states are obtained from the above polaronic states by applying spin angular momentum ladder operator $S^{-}=\Sigma_{i} s_{i}^{-}$where " $i$ " runs over all $\pi$ orbitals used for describing the conjugated molecules. $\psi_{\mathrm{e}-\mathrm{hR}}^{|1 / 2,1 / 2\rangle}(0)$ is evolved using the total Hamiltonian of the system described as

$$
H_{\mathrm{tot}}=H_{M^{+}}^{\mathrm{PPP}}+H_{M^{-}}^{\mathrm{PPP}}+H_{P}+H_{\mathrm{int}} .
$$

The wave-packet $\psi_{\mathrm{e}-\mathrm{hR}}(0)$ is time evolved using techniques described in the previous section. As time elapses, the oppo- 
TABLE II. Vertical singlet and triplet excited states for polyenes of varying sizes. Here we also present the oscillator strengths connecting the singlet excited states to the ground state. We have listed only those triplet states whose energies are below the energy of the highest excited singlet state quoted plus $1.5 \mathrm{eV}$.

\begin{tabular}{cccc}
\hline \hline $\begin{array}{c}\text { Size } \\
\text { of polyenes }\end{array}$ & $\begin{array}{c}\text { Singlet gaps } \\
\text { from } S_{0} \\
(\mathrm{eV})\end{array}$ & $\begin{array}{c}\text { Oscillator } \\
\text { strength }\end{array}$ & $\begin{array}{c}\text { Triplet gaps } \\
\text { from } S_{0} \\
(\mathrm{eV})\end{array}$ \\
\hline 2 & 7.09 & 3.61 & 3.73 \\
& 10.82 & 0.00 & \\
4 & 5.47 & 0.00 & 2.75 \\
& 5.81 & 6.89 & 4.21 \\
& & & 6.73 \\
6 & & & 2.27 \\
& & 0.00 & 3.50 \\
& 4.50 & 9.87 & 4.38 \\
& 5.06 & & 5.64 \\
& & & 6.43 \\
& & & 2.01 \\
& 3.92 & 0.00 & 2.99 \\
& 4.59 & 12.75 & 3.88 \\
& & & 4.47 \\
& & & 4.89 \\
& & & 5.72 \\
\hline \hline
\end{tabular}

sitely charged polyenes recombine to form excited singlet or triplet excitons on one molecule and a singlet ground state on the other. Such recombination process as described in Eq. (21) is mediated by the paramagnetic impurity through the exchange and CT pathway.

$$
M^{+}+M^{-} \rightarrow G+S / T \text {. }
$$

In order to follow the time evolution of the product states in the charge recombination phenomenon, the evolved state $\psi_{\mathrm{e}-\mathrm{hR}}(t)$ is projected on to the direct product of the excited singlet (singlet pathway) or triplet (triplet pathway) states, the singlet ground state and the paramagnetic state. The final states in the singlet and the triplet pathways are given by

$$
\begin{aligned}
\psi_{\mathrm{e}-\mathrm{hR}}^{T} & =\frac{1}{\sqrt{3}}|1,0\rangle_{T_{m}} \otimes|0,0\rangle_{G} \otimes\left|\frac{1}{2}, \frac{1}{2}\right\rangle_{P}-\frac{2}{\sqrt{3}}|1,1\rangle_{T_{m}} \\
& \otimes|0,0\rangle_{G} \otimes\left|\frac{1}{2},-\frac{1}{2}\right\rangle_{P}, \\
\psi_{\mathrm{e}-\mathrm{hR}}^{S} & =|0,0\rangle_{S_{m}} \otimes|0,0\rangle_{G} \otimes\left|\frac{1}{2}, \frac{1}{2}\right\rangle_{P},
\end{aligned}
$$

where $|1,1\rangle_{T_{m}}$ represents the $m$ th $S=1$ and $M_{s}=1$ eigenstates of the polyene Hamiltonian expressed as a linear combination of the triplet VB functions. $|1,0\rangle_{T_{m}}$ is obtained by operating $S^{-}$on the $|1,1\rangle_{T_{m}}$ as in the ISC process. Similarly the $|0,0\rangle_{S_{m}}$ state represents the $m$ th $S=0$ and $M_{s}=0$ eigenstates of the polyene Hamiltonian. The total spin and its corresponding z-component of the final states in both pathways are taken to be $1 / 2$. The coefficients of these states in the time evolved packets are given by

$$
\begin{aligned}
& C_{T_{m}}=\left\langle\psi_{\mathrm{e}-\mathrm{hR}}(t) \mid \psi_{\mathrm{e}-\mathrm{hR}}^{T}\right\rangle, \\
& C_{S_{m}}=\left\langle\psi_{\mathrm{e}-\mathrm{hR}}(t) \mid \psi_{\mathrm{e}-\mathrm{hR}}^{S}\right\rangle .
\end{aligned}
$$

The probability that at time " $t$ " the polaron recombines pursuing the triplet or the singlet pathway resulting in the formation of the $m$ th excited triplet or $m$ th singlet state is given by

$$
P_{T_{m} / S_{m}}^{\mathrm{e}-\mathrm{hR}}(t)=\left|C_{T_{m} / S_{m}}\right|^{2} .
$$

The $P_{T_{m} / S_{m}}^{\mathrm{e}-\mathrm{hR}}$ is followed as a function of time upto $66 \mathrm{fs}$ for several low-lying singlet and triplet states of the polyene.

\begin{tabular}{|c|c|c|c|c|c|c|c|}
\hline \multirow[b]{2}{*}{$\begin{array}{c}\text { Size } \\
\text { of polyene }\end{array}$} & \multirow[b]{2}{*}{$\begin{array}{c}\text { Site energies } \\
\text { of paramagnetic site } \\
(\mathrm{eV})\end{array}$} & \multicolumn{3}{|c|}{$2 \mathrm{~A}_{\mathrm{g}}$ initial state } & \multicolumn{3}{|c|}{$1 \mathrm{~B}_{\mathrm{u}}$ initial state } \\
\hline & & Total yield & $\begin{array}{c}\text { Yield } \\
\text { of lowest } \\
\text { triplet state }\end{array}$ & $\begin{array}{c}\text { Highest } \\
\text { triplet yield }\end{array}$ & Total yield & $\begin{array}{c}\text { Yield } \\
\text { of lowest } \\
\text { triplet state }\end{array}$ & $\begin{array}{l}\text { Highest } \\
\text { triplet yield }\end{array}$ \\
\hline \multirow[t]{3}{*}{2} & 2.0 & $1.82 \times 10^{-3}$ & $\cdots$ & $\cdots$ & $3.74 \times 10^{-2}$ & $\cdots$ & $\cdots$ \\
\hline & 3.0 & $1.35 \times 10^{-3}\left\{3.74 \times 10^{-2}\right\}$ & $\cdots$ & $\cdots$ & $6.30 \times 10^{-3}\left\{9.53 \times 10^{-2}\right\}$ & $\cdots$ & $\cdots$ \\
\hline & 5.0 & $6.25 \times 10^{-3}\left\{2.01 \times 10^{-2}\right\}$ & $\cdots$ & $\cdots$ & $9.99 \times 10^{-3}\left\{9.91 \times 10^{-2}\right\}$ & $\cdots$ & $\cdots$ \\
\hline \multirow[t]{3}{*}{4} & 2.0 & 0.23 & $8.61 \times 10^{-3}$ & $0.21(3)$ & 0.12 & $6.64 \times 10^{-3}$ & $5.96 \times 10^{-2}(2)$ \\
\hline & 3.0 & $0.40\{0.08\}$ & $2.13 \times 10^{-2}$ & $0.34(3)$ & $0.27\{0.08\}$ & $4.76 \times 10^{-2}$ & $0.18(3)$ \\
\hline & 5.0 & $0.25\{0.41\}$ & $2.68 \times 10^{-3}$ & $0.17(2)$ & $0.07\{0.15\}$ & $3.88 \times 10^{-3}$ & $3.78 \times 10^{-2}(3)$ \\
\hline \multirow[t]{3}{*}{6} & 2.0 & 0.13 & $8.63 \times 10^{-3}$ & $5.29 \times 10^{-2}(2)$ & 0.13 & $8.75 \times 10^{-3}$ & $5.84 \times 10^{-2}(2)$ \\
\hline & 3.0 & $4.96\{0.97\}$ & 1.41 & $2.73(3)$ & $0.60\{0.26\}$ & 0.12 & $0.26(3)$ \\
\hline & 5.0 & $0.57\{0.49\}$ & 0.11 & $0.22(3)$ & $0.33\{0.05\}$ & $6.58 \times 10^{-2}$ & $8.81 \times 10^{-2}(3)$ \\
\hline \multirow[t]{3}{*}{8} & 2.0 & $4.28 \times 10^{-2}$ & $5.26 \times 10^{-3}$ & $9.33 \times 10^{-3}$ & $3.87 \times 10^{-2}$ & $2.97 \times 10^{-3}$ & $1.10 \times 10^{-2}(2)$ \\
\hline & 3.0 & $9.49 \times 10^{-2}\left\{1.08 \times 10^{-2}\right\}$ & $1.84 \times 10^{-2}$ & $1.92 \times 10^{-2}(2)$ & $0.12\left\{6.44 \times 10^{-3}\right\}$ & $1.53 \times 10^{-2}$ & $2.55 \times 10^{-2}(5)$ \\
\hline & 5.0 & $0.30\left\{2.87 \times 10^{-3}\right\}$ & $4.67 \times 10^{-2}$ & $9.36 \times 10^{-2}(5)$ & $0.12\left\{1.46 \times 10^{-3}\right\}$ & $1.85 \times 10^{-2}$ & $2.29 \times 10^{-2}(2)$ \\
\hline
\end{tabular}
Time integrated yields in both the channels are given by

$$
I_{T / S}=\sum_{m} \int_{0}^{t_{\max }} P_{T_{m} / S_{m}}^{\mathrm{e}-\mathrm{hR}}(t) d t
$$

TABLE III. Triplet yields for ISC starting from low-lying singlet excited states for different polyenes in presence of the paramagnetic impurity with one orbital. Results for a paramagnetic site with two orbitals are given in curly brackets, in the total yield columns. The integers in parentheses in the column for the highest yield for $A_{g} / B_{u}$ state give the quantum number of the triplet state to which this highest yield corresponds. 

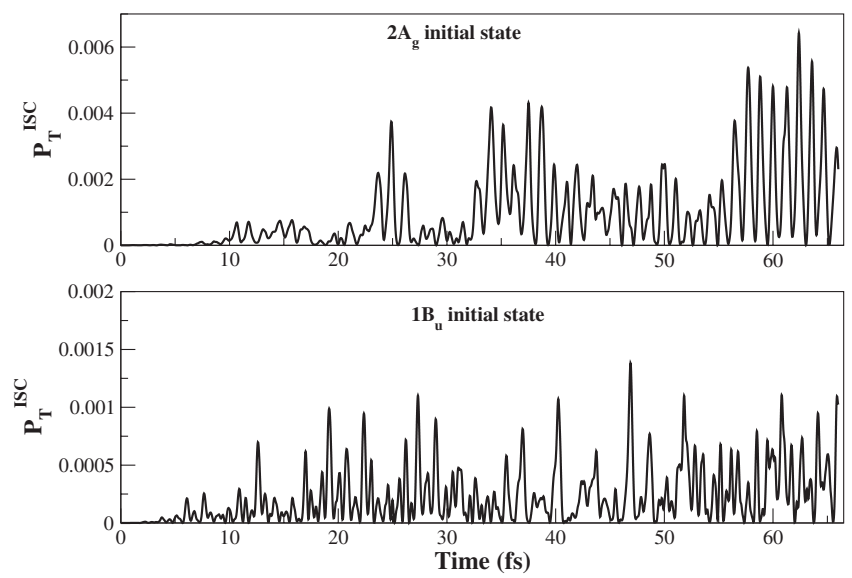

FIG. 4. Time evolution of the probability of the most favorable triplet formed in the ISC process starting from $2 \mathrm{~A}_{\mathrm{g}}$ (top) and $1 \mathrm{~B}_{\mathrm{u}}$ (bottom) states for octatetraene molecule in presence of a paramagnetic impurity with unpaired electron occupying a single orbital. The quantum numbers of the final triplet state for the top panel is 5 and the bottom panel is 2 which correspond to the states producing highest yield (Table II).

\section{Wave-packet dynamics for TQ study}

Similar to the previous processes the initial wave-packet for the TQ process is given by a direct product of the lowlying triplet states $\left(T_{m}\right)$ of the molecule and the ground state triplet of the oxygen molecule $\left(T_{\mathrm{O}_{2}}\right)$. There are two pathways for TQ, namely, the triplet pathway and the singlet pathway; the corresponding wave-packets are given by

$$
\begin{aligned}
\Psi_{\mathrm{TQ}}^{T}(0)= & \sqrt{\frac{1}{2}}|1,-1\rangle_{\mathrm{O}_{2}} \otimes|1,1\rangle_{T_{m}} \\
& -\sqrt{\frac{1}{2}}|1,1\rangle_{\mathrm{O}_{2}} \otimes|1,-1\rangle_{T_{m}}, \\
\Psi_{\mathrm{TQ}}^{S}(0)= & \sqrt{\frac{1}{3}}|1,-1\rangle_{\mathrm{O}_{2}} \otimes|1,1\rangle_{T_{m}}-\sqrt{\frac{1}{3}}|1,0\rangle_{\mathrm{O}_{2}} \\
& \otimes|1,0\rangle_{T_{m}}+\sqrt{\frac{1}{3}}|1,1\rangle_{\mathrm{O}_{2}} \otimes|1,-1\rangle_{T_{m}},
\end{aligned}
$$

where, $|1,1\rangle_{T_{m}}$ represents the $m$ th $S=1$ and $M_{S}=1$ eigen- state of the polyene Hamiltonian expressed as a linear combination of the triplet VB functions and $|1,1\rangle_{\mathrm{O}_{2}}$ is the triplet ground state of oxygen molecule. $|1,0\rangle_{T_{m}} / \mathrm{O}_{2}$ and $|1,-1\rangle_{T_{m} / \mathrm{O}_{2}}$ are obtained by successively operating $S^{-}$on $|1,1\rangle_{T_{m} / \mathrm{O}_{2}}$ as in the previous cases. $\Psi_{\mathrm{TQ}}^{S}$ and $\Psi_{\mathrm{TQ}}^{T}$ are evolved using the total Hamiltonian of the system given by

$$
H_{\text {tot }}=H_{\text {polymer }}^{\text {PPP }}+H_{\mathrm{O}_{2}}+H_{\text {int }} .
$$

The $\Psi_{\mathrm{TQ}}^{S / T}(t)$ is then projected on to the direct product of the singlet ground state of the molecular Hamiltonian and the triplet or singlet state of the oxygen molecule. The final states for the projection are given by $\left|\Psi_{\mathrm{TQ}}\left(S_{0}, T\right)\right\rangle=\left|S_{0}\right\rangle$ $\otimes|1,0\rangle_{\mathrm{O}_{2}}$ and $\left|\Psi_{\mathrm{TQ}}\left(S_{0}, S\right)\right\rangle=\left|S_{0}\right\rangle \otimes|0,0\rangle_{\mathrm{O}_{2}}$, respectively. The coefficients of these states in the time evolved wave-packet are given by

$$
\begin{aligned}
& C_{S_{0}, S}(t)=\left\langle\Psi_{\mathrm{TQ}}\left(S_{0}, S\right) \mid \Psi_{\mathrm{TQ}}^{S}(t)\right\rangle, \\
& C_{S_{0}, T}(t)=\left\langle\Psi_{\mathrm{TQ}}\left(S_{0}, T\right) \mid \Psi_{\mathrm{TQ}}^{T}(t)\right\rangle .
\end{aligned}
$$

Equation (26) takes into account the total spin (spin one in case of triplet path and spin zero in case of singlet path) and the total Hamiltonian being nonrelativistic conserves this spin value. However, the quenching of the triplet state is possible in presence of paramagnetic ground state of oxygen in different $S_{z}$ sectors $\left(|1,1\rangle_{\mathrm{O}_{2}},|1,0\rangle_{\mathrm{O}_{2}},|1,-1\rangle_{\mathrm{O}_{2}}\right)$ by conserving the total spin. The probability at time $t$ that the polyene triplet is quenched to its singlet ground state either through singlet $\left(P_{S_{0}, S}^{\mathrm{TQ}}\right)$ or triplet $\left(P_{S_{0}, T}^{\mathrm{TQ}}\right)$ route is given by

$$
P_{S_{0}, S / T}^{\mathrm{TQ}}(t)=\left|C_{S_{0}, S / T}(t)\right|^{2} .
$$

The $P_{S_{0}, S / T}^{\mathrm{TQ}}(t)$ is followed as a function of time up to

\begin{tabular}{|c|c|c|c|c|c|c|c|}
\hline \multirow[b]{2}{*}{$\begin{array}{c}\text { Size } \\
\text { of polyene }\end{array}$} & \multirow[b]{2}{*}{$\begin{array}{c}\text { Site energies } \\
\text { of paramagnetic } \\
\text { site }\end{array}$} & \multicolumn{3}{|c|}{$\begin{array}{l}\text { Total triplet yield } \\
\text { from } 2 \mathrm{~A}_{\mathrm{g}} \text { state }\end{array}$} & \multicolumn{3}{|c|}{$\begin{array}{l}\text { Total triplet yield } \\
\text { from } 1 \mathrm{~B}_{\mathrm{u}} \text { state }\end{array}$} \\
\hline & & $\begin{array}{l}\text { System with electron } \\
\text { withdrawing } \\
\text { substituents }\end{array}$ & $\begin{array}{l}\text { Unsubstituted } \\
\text { system }\end{array}$ & $\begin{array}{c}\text { System with electron } \\
\text { donating } \\
\text { substituents }\end{array}$ & $\begin{array}{l}\text { System with electron } \\
\text { withdrawing } \\
\text { substituents }\end{array}$ & $\begin{array}{l}\text { Unsubstituted } \\
\text { system }\end{array}$ & $\begin{array}{c}\text { System with electron } \\
\text { donating } \\
\text { substituents }\end{array}$ \\
\hline \multirow[t]{2}{*}{2} & 3.0 & $2.32 \times 10^{-3}$ & $1.35 \times 10^{-3}$ & $1.18 \times 10^{-3}$ & $5.34 \times 10^{-3}$ & $6.30 \times 10^{-3}$ & $1.10 \times 10^{-2}$ \\
\hline & 5.0 & $2.27 \times 10^{-3}$ & $6.25 \times 10^{-3}$ & $4.23 \times 10^{-2}$ & $5.36 \times 10^{-3}$ & $9.99 \times 10^{-3}$ & $1.61 \times 10^{-2}$ \\
\hline \multirow[t]{2}{*}{4} & 3.0 & 0.23 & 0.40 & 2.68 & 0.23 & 0.27 & 1.01 \\
\hline & 5.0 & 0.05 & 0.25 & 0.12 & 0.03 & 0.07 & 0.24 \\
\hline \multirow[t]{2}{*}{6} & 3.0 & 0.42 & 4.96 & 0.30 & 0.13 & 0.60 & 0.12 \\
\hline & 5.0 & 0.45 & 0.57 & 2.27 & 0.38 & 0.33 & 0.39 \\
\hline \multirow[t]{2}{*}{8} & 3.0 & 0.19 & 0.09 & 0.11 & 0.19 & 0.12 & 0.06 \\
\hline & 5.0 & 0.24 & 0.30 & 0.31 & 0.23 & 0.12 & 0.20 \\
\hline
\end{tabular}
$t_{\max }=66 \mathrm{fs}$ for several low-lying triplet states of the polyene. Time integrated yields in both the channels (singlet and triplet final states of the oxygen molecule) are given by

$$
I_{S_{0}, S / T}=\sum_{m} \int_{0}^{t_{\max }} P_{S_{0}, S / T}^{\mathrm{TQ}}(t) d t
$$

TABLE IV. Triplet yields starting from low-lying singlet excited states $\left(2 \mathrm{~A}_{\mathrm{g}}\right.$ and $\left.1 \mathrm{~B}_{\mathrm{u}}\right)$ of substituted and unsubstituted polyenes of different lengths in the presence of paramagnetic impurity with one orbital. 
TABLE V. Singlet and triplet yields in the charge recombination process in the presence as well as the absence of paramagnetic impurity.

\begin{tabular}{|c|c|c|c|c|}
\hline \multirow{2}{*}{$\begin{array}{l}\text { Site energies } \\
\text { of paramagnetic site } \\
(\mathrm{eV})\end{array}$} & \multicolumn{2}{|c|}{ Triplet yield } & \multicolumn{2}{|c|}{ Singlet yield } \\
\hline & $\begin{array}{l}\text { Degenerate } \\
\text { orbital }\end{array}$ & $\begin{array}{l}\text { Nondegenerate } \\
\text { orbital }\end{array}$ & $\begin{array}{l}\text { Degenerate } \\
\text { orbital }\end{array}$ & $\begin{array}{c}\text { Nondegenerate } \\
\text { orbital }\end{array}$ \\
\hline 3.0 & 1.26 & 0.06 & 0.51 & 0.57 \\
\hline 5.0 & 1.28 & 0.09 & 0.52 & 0.75 \\
\hline Without paramagnetic impurity & \multicolumn{2}{|c|}{0.29} & \multicolumn{2}{|c|}{0.94} \\
\hline
\end{tabular}

\section{RESULTS AND DISCUSSIONS}

Before we focus on the time dependent calculations, we turn our attention to the calculation of excitation spectrum of the polyene systems. Here, we have performed our calculations for four oligomers of polyenes, namely, ethylene, butadiene, 1,3,5-hexatriene, and 1,3,5,7-octatetraene. For these systems, the lowest dipole allowed singlet, the lowest dipole forbidden singlet and some triplet excited states are listed in Table II.

As is evident from Table II, the first excited state is dipole forbidden from the ground state except for the ethylene molecule. On the contrary, the second excited states in these polyenes are dipole allowed and its oscillator strength increases linearly as we increase the size of the system. The optical gap is $7.09 \mathrm{eV}$ in ethylene molecule and the gap reduces to $3.92 \mathrm{eV}$ in 1,3,5,7-octatetraene. Similarly the singlet triplet gap is $3.73 \mathrm{eV}$ in ethylene and reduces to $2.01 \mathrm{eV}$ for octatetraene. Another interesting feature is that the energies of dipole forbidden singlet states are approximately twice that of the lowest triplet states as is to be expected from the description of the $2 \mathrm{~A}_{\mathrm{g}}$ state as being composed of the triplets. ${ }^{33}$

\section{A. Results of the ISC studies}

In conjugated polymers, the photophysics is mainly dominated by the low-lying singlet and triplet excited states. Furthermore, in most of these organic materials, irrespective of the mode of excitations, luminescence almost always occurs from the lowest singlet excited state as is known from Kasha's rule. ${ }^{32}$ For these reasons, we consider the two lowlying singlet excited states as starting states for ISC process. Our choice is also motivated by the different symmetries of these states which is evident from the magnitude of the oscillator strengths listed in Table II. In the triplet manifold, we take into account only those triplet states whose energies are not higher than the singlet states considered, by more than $\sim 1.5 \mathrm{eV}$. Such a consideration leaves us with three, five, and seven low-lying triplet states in butadiene, hexatriene, and octatetraene, respectively, for consideration. The time integrated yields listed in Table III, are obtained by integrating the time dependent probability [Eqs. (16), (24), and (29)] over a finite period of time (66 fs). From the table below, we see that the intersystem crossing yields are sensitive to the site energies as well as number of orbitals on the paramagnetic site. The state to which the highest yield is found also depends sensitively on the initial state as well as the site energy of the impurity orbital. Since the electron-hole sym- metry for the full system is broken due to different site energies, we cannot involve any symmetry arguments. In most cases, the conversion to the triplet is larger from $2 \mathrm{~A}_{\mathrm{g}}$ state rather than the $1 \mathrm{~B}_{\mathrm{u}}$ state; the only major exception being the ethylene molecule. This can be attributed to the fact that $2 \mathrm{~A}_{\mathrm{g}}$ state is mainly dominated by the spin wave excitation over the antiferromagnetic ground state whereas the charge transfer states dominate the $1 \mathrm{~B}_{\mathrm{u}}$ state. Thus the $2 \mathrm{~A}_{\mathrm{g}}$ state can be thought of as a pair of triplets ${ }^{33}$ which results in higher triplet yields compared to $1 \mathrm{~B}_{\mathrm{u}}$ state. The conversion rates also depend on the magnitude of the interaction matrix elements between initial and final states and also on the inverse of the energy difference between the same as can be discerned from perturbation theory. ${ }^{34}$ Yield of the triplets through ISC from a singlet state $\left(2 \mathrm{~A}_{\mathrm{g}} / 1 \mathrm{~B}_{\mathrm{u}}\right)$ is determined by the strength of the exchange integrals (direct pathway) and the transfer integrals (CT pathway) present between the polyene and the paramagnetic site which constitute the total Hamiltonian (Fig. 2). It is worth remarking that in the case of hexatriene, for $\varepsilon$ $=3.0 \mathrm{eV}$, there is a large ISC cross section. This could be because of the near resonance between the initial and final states, which has a large intermolecular interaction matrix element. In addition to the time integrated yields listed in Table III, we also present the time evolution of the ISC process for the octatetraene molecule in Fig. 4.

To mimic the effect of keto-defects on the polyfluorenes, we vary the site energies of carbon orbitals on the polyene chain neighboring the paramagnetic metal. For electron donating substituent, we increase the site energies of the carbon

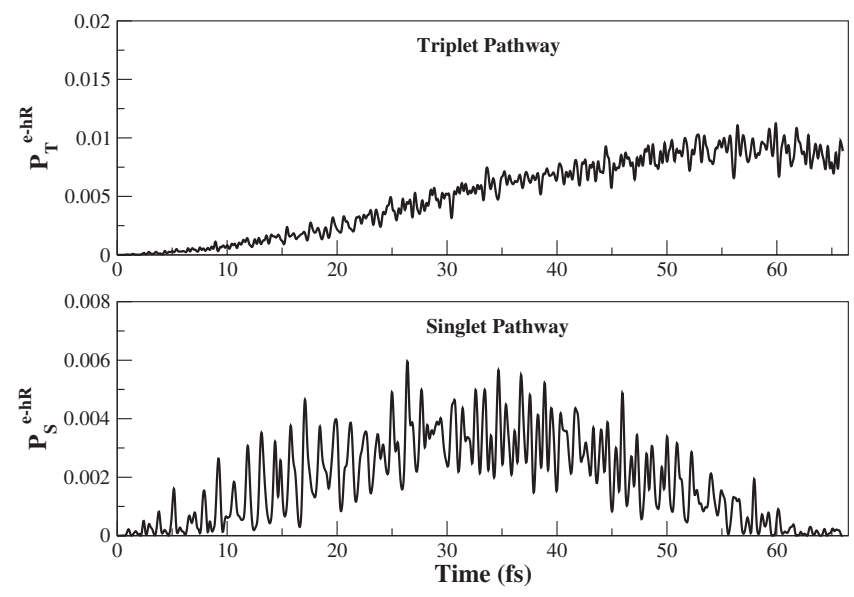

FIG. 5. Time evolution of $P_{S / T}^{\mathrm{e}-\mathrm{hR}}$ in e-hR process in the presence of paramagnetic impurity with one unpaired electron occupying doubly degenerate orbitals. The quantum number for the final states in triplet (top) and singlet (bottom) pathways is 6 . 

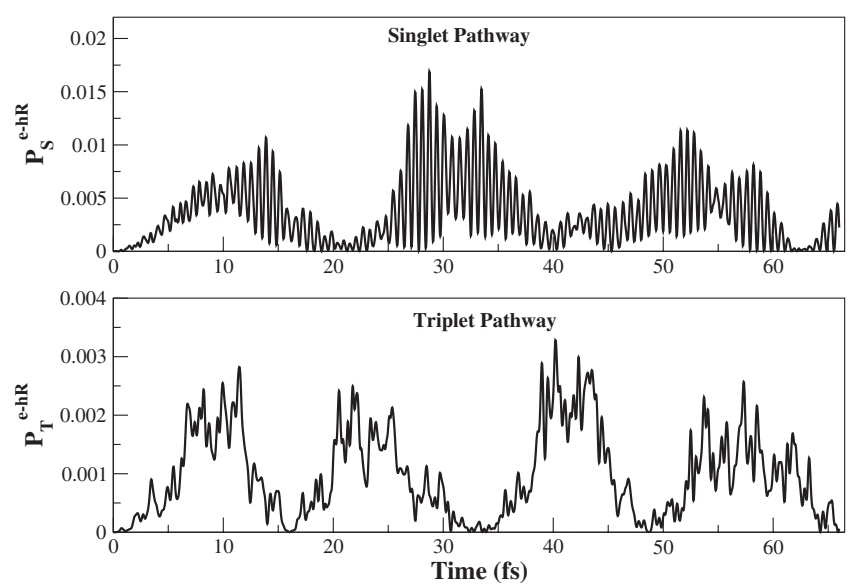

FIG. 6. Time evolution of e-hR process in absence of paramagnetic impurity. The quantum numbers for the final states in singlet (top) and triplet (bottom) pathways are 4 and 3, respectively.

orbitals on polyene chain neighboring to the paramagnetic metal by $0.5 \mathrm{eV}$, whereas for electron withdrawing substituent we decrease the site energies by the same amount.

Table IV clearly shows that there is sufficient ISC process leading to triplet formation even in the polyenes which mimic the keto-defect. The triplet yields are slightly higher for electron donating groups rather than electron withdrawing substituents. In order to estimate the triplet yields through the charge transfer and the exchange routes we have reduced the transfer integrals $\left(t_{i j}^{\prime}\right)$ by an order of magnitude keeping the exchange integrals constant. Such reductions in transfer integrals between the polyene and metal sites result in negligible triplet formation which points to the dominance of the CT mechanism over the exchange pathway in ISC process.

\section{B. e-hR in the presence of magnetic impurity}

We have performed our calculation of charge recombination by two butadiene units placed at $3.0 \AA$ apart along the $x$-axis in the presence of a paramagnetic atom placed at a distance of $4 \AA$ along the $y$-axis from the center of the two butadiene units (Fig. 3). The site energies of the paramagnetic impurity is taken to be 3.0 and $5.0 \mathrm{eV}$ for reasons discussed earlier. The Hubbard $U$ is taken to be same as that used in the ISC process mediated by paramagnetic impurity. Calculated yields in both singlet and triplet channels are shown in Table V. Here we have also presented the total yield for a paramagnetic moiety with two degenerate orbitals. For the sake of comparison, we also present in Table V the yields of singlets and triplets formed due to direct charge recombination (in absence of metal impurity).

From the above table it is evident that the singlets are more favored if the unpaired electron of the paramagnetic impurity is restricted to a single orbital. For a spin in a degenerate pair of orbitals, although the yields of singlets roughly remain the same, the triplet yield is 2.5 times higher than the singlet yield. So the charge recombination process has higher triplet yields in presence of paramagnetic metal impurities with unpaired electron in a doubly degenerate set of orbitals. Furthermore, such charge recombination process
TABLE VI. Yields in TQ process starting from the lowest triplet state of polyenes of different lengths. We have also varied the site energies of the paramagnetic metal impurity in these calculations.

\begin{tabular}{cccc}
\hline \hline & & \multicolumn{2}{c}{$\begin{array}{c}\text { Yield of ground sate } \\
\text { from lowest triplet state }\end{array}$} \\
\cline { 3 - 4 } $\begin{array}{c}\text { Size } \\
\text { of polyenes }\end{array}$ & $\begin{array}{c}\text { Site energies } \\
\text { of paramagnetic site } \\
(\mathrm{eV})\end{array}$ & $\begin{array}{c}\text { Degenerate } \\
\text { orbital }\end{array}$ & $\begin{array}{c}\text { Nondegenerate } \\
\text { orbital }\end{array}$ \\
\hline 2 & 3.0 & 0.28 & $5.36 \times 10^{-3}$ \\
& 5.0 & 1.06 & $2.00 \times 10^{-3}$ \\
4 & 3.0 & $3.76 \times 10^{-2}$ & $7.88 \times 10^{-3}$ \\
& 5.0 & $1.48 \times 10^{-2}$ & $4.11 \times 10^{-3}$ \\
6 & 3.0 & $1.87 \times 10^{-2}$ & $1.86 \times 10^{-2}$ \\
8 & 5.0 & $4.72 \times 10^{-3}$ & $2.29 \times 10^{-2}$ \\
& 3.0 & $1.09 \times 10^{-2}$ & $1.46 \times 10^{-2}$ \\
& 5.0 & $1.82 \times 10^{-2}$ & $5.03 \times 10^{-2}$ \\
\hline \hline
\end{tabular}

in presence of a site with doubly degenerate orbitals yields higher triplets than the ISC pathway; except near resonance as with hexatriene with $\varepsilon=3.0 \mathrm{eV}$ (Table III). As shown in case of ISC process, we present in Figs. 5 and 6 the probability $\left(P_{S_{m} T_{m}}^{\mathrm{e}-\mathrm{TR}}\right)$ of charge recombination for the triplet and singlet pathways as a function of time both in the presence and in the absence of a paramagnetic impurity.

As evident from Fig. 5, the yield of the triplets is much larger compared to the singlets. Furthermore, Fig. 5 also shows that the time integrated triplet yields are larger than the singlet yields. We have evolved both the channels for 66 fs time regime for the sake of comparison with those obtained from the other time dependent processes. Now we turn our attention to the time evolution of e-hR process in absence of paramagnetic impurity (Fig. 6). Figure 6 shows the expected dominance of the singlet pathway in the e-hR process in absence of the magnetic impurity. It is also interesting to see that the probability is approximately periodic and is similar to Rabi oscillations in a single two-level system. ${ }^{35}$ If coupling to the vibrational degrees is included then the oscillation will be damped due to an irreversible shift of population from the initial state to the continuum bath states provided by the vibrations. ${ }^{34,36,37}$
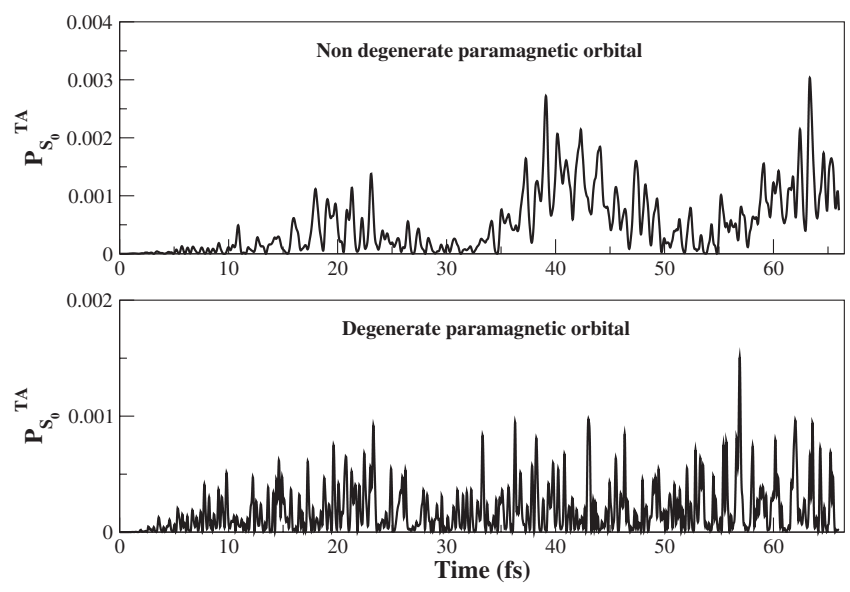

FIG. 7. Time evolution of triplet annihilation process for octatetraene molecule in the presence of paramagnetic impurity with non degenerate (top) and doubly degenerate (bottom) orbitals. The site energy of the paramagnetic orbital is chosen to be $5.0 \mathrm{eV}$. 
TABLE VII. TQ yields through triplet channels for substituted and unsubstituted polyenes. These yields are calculated from the lowest triplet states of polyenes of different sizes.

\begin{tabular}{lccc}
\hline \hline & \multicolumn{3}{c}{ Quenching yields } \\
\cline { 2 - 4 } Polyene systems & Electron withdrawing substituent & No substituent & Electron donating substituent \\
\hline Ethylene & $4.20 \times 10^{-2}$ & $4.83 \times 10^{-2}$ & $5.77 \times 10^{-2}$ \\
Butadiene & $1.32 \times 10^{-3}$ & $1.57 \times 10^{-2}$ & $1.38 \times 10^{-3}$ \\
$1,3,5$ hexatriene & $5.96 \times 10^{-2}$ & $9.77 \times 10^{-2}$ & $6.18 \times 10^{-2}$ \\
\hline \hline
\end{tabular}

Experimental results are well explained by these theoretical observations. First the intensity of the $500 \mathrm{~nm}$ EL peak increases drastically in presence of Pd, Ir, and $\mathrm{Re}^{38,39}$ complexes (which have unpaired electron in degenerate orbitals) as dopant but not as much when doped with $\mathrm{Cu}$ (II) salts (which has an unpaired electron in a nondegenerate orbital). ${ }^{11}$ Second PL does not yield much triplets, clearly depicting the inefficiency of ISC process compared to the charge recombination phenomenon. Besides, the presence of small concentration of triplets in polyfluorene films (in absence of metal impurities) in EL can also be attributed to the charge recombination as indicated by our theoretical investigations.

\section{Triplet annihilation in the presence of metal impurity and oxygen}

Here we focus on the quenching of triplets in the presence of paramagnetic metal impurity. This process nullifies the effect of ISC process even in the absence of other quenchers like oxygen and nitric-oxide. Here we restrict our calculations only to the quenching rates of lowest triplet state of polyenes. In Table VI we present the quenching rates of triplets under various conditions.

The TQ rates are high in ethylene, the shortest polyene molecule for degenerate paramagnetic impurity. The TQ yields in presence of paramagnetic impurity with nondegenerate orbital increase with the system sizes. In addition to time integrated yields, we present in Fig. 7 the time evolution of the triplet annihilation probability for the octatetraene molecule with paramagnetic impurity having $\varepsilon=5.0 \mathrm{eV}$.

Such TQ process will reduce the yields of triplets formed during the ISC process and clearly underlines the adverse effect of Pd on PL spectrum, as observed experimentally. In air however, this TQ process is reinforced by the presence of paramagnetic oxygen molecule.

The calculations for TQ in presence of oxygen have been conducted for ethylene, butadiene, 1,3,5-hexatriene molecules. Since we consider four active orbitals for the oxygen molecule, the Hilbert space of the Hamiltonian becomes too large to deal with 1,3,5,7 octatetraene. However, we have evolved this system for a shorter period of time and verified that the general conclusions we arrive at from calculations up to hexatriene system are vindicated. The oxygen parameters are fixed as discussed earlier in Sec. III. The yields calculated by quenching the lowest triplet state of the polyenes in presence of oxygen molecule in which the final state of the oxygen molecule is the ground state triplet (triplet channel), are shown in Table VII. ${ }^{40}$ The quenching yield through the singlet channel, in which the final state of the oxygen molecule is the lowest singlet state, is almost negligible. If we consider the free spin statistics limit, the triplet channel yields should be higher than the singlet channel by a factor of three. But this statistics is violated in the presence of interactions $^{41}$ as known from the study of various other processes like e-hR, and triplet-triplet annihilation. ${ }^{42,43}$ We have also presented the TQ yields for substituted polyenes in Table VII.

The TQ yields do not vary significantly on substituting the polyenes with either electron donor or acceptor groups. We may therefore conclude that the quenching rates for triplet excitons trapped at defect sites or untrapped are quite similar. Similar to ISC process, we have also studied the importance of exchange mechanism with respect to the charge transfer pathway by reducing the magnitude of transfer integrals between the oxygen molecule and the polyene but keeping the two electron integrals the same. Quite unlike the formation of triplets by ISC, the TQ yield increases by an order of magnitude, clearly suggesting the dominance of exchange pathways over CT route in quenching of triplets. Finally we present the time evolution of the TQ process for the 1,3,5-hexatriene molecule in Fig. 8.

\section{SUMMARY AND CONCLUSION}

The strikingly different PL and EL of polyfluorene films as well as the nature of EL maxima around $500 \mathrm{~nm}$ motivated the present study. PL and EL spectra generally coincide in the organic solid state both for molecules and conjugated polymers. In this respect, the PF system is exceptional. The PL in solution and in the solid state yield similar spectra,

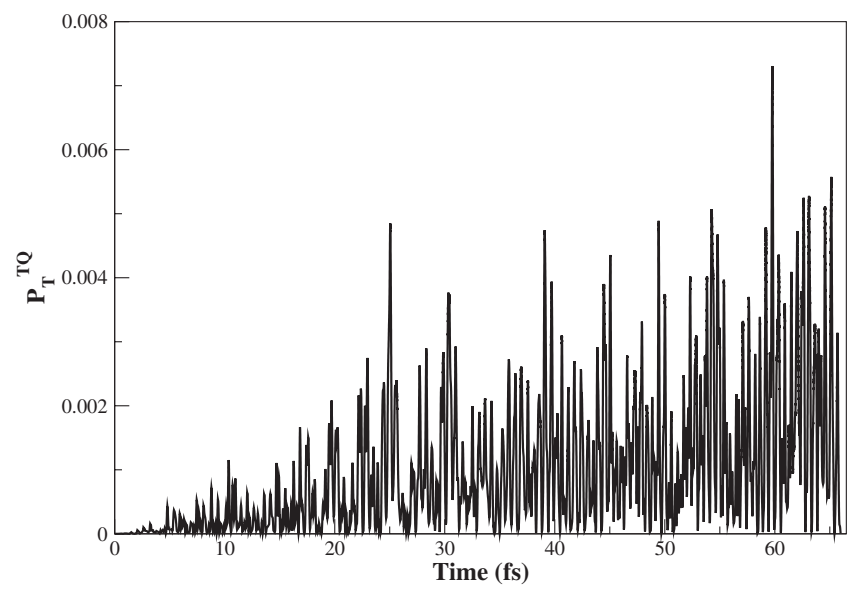

FIG. 8. Time evolution of the TQ process for 1,3,5-hexatriene molecule in the presence of paramagnetic oxygen. 
both show a maximum around $412-430 \mathrm{~nm}$ with a shoulder at $500 \mathrm{~nm}$. The EL spectrum in the presence of oxygen is similar to the PL spectrum. However, in nitrogen atmosphere much of the spectral intensity shifts to the $500 \mathrm{~nm}$ peak. We assign the $500 \mathrm{~nm}$ peak seen in EL in nitrogen atmosphere to the triplet state of the polymer. In EL experiments, geminate recombination of polaronic pairs result in formation of both singlet and triplet excitons. The concentration of the triplet excitons would be high in this case especially in presence of paramagnetic impurity with degenerate magnetic orbitals. Evidence for triplet emission as the origin of the $500 \mathrm{~nm}$ peak comes from studying polymers doped with paramagnetic salts. In the presence of heavy metal salts or complexes of transition metal such as Ir, Pd, and Re, there is considerable enhancement of the intensity of the $500 \mathrm{~nm}$ peak; this enhancement is seen to be much lower with paramagnetic salts of $\mathrm{Cu}$ (II). Transition metals are known to help in spin crossover processes. Such spin catalysis is mostly localized on the metal site. Such processes are known not only for polyfluorenes but also for other polymers like PPV (Ref. 44) etc.

Our theoretical studies show that the excited singlet state can be converted efficiently to a triplet state by paramagnetic impurities due to electron-electron interactions. Furthermore, such triplet formation is also possible from charge recombination process which is the dominant mechanism for EL; thus we can have a large concentration of triplets in the presence of paramagnetic impurities in an EL process. This theoretical result is also substantiated by experimental observations. ${ }^{45}$ Our study employs model parameters which are consistent with UVPES data for $4 d$ and $5 s$ orbitals of metals like Pd, Re, and Ir. Our study also shows that if the unpaired electron in the paramagnetic ion or atom is located in a degenerate set of orbitals then the formation of triplets by charge recombination process is highly efficient. This explains why salts of Ir, Pd, and Re lead to more intense 500 $\mathrm{nm}$ peak than the salts of $\mathrm{Cu}$ (II) complexes in EL experiments.

Our study also explains the absence of the $500 \mathrm{~nm}$ EL peak in $\mathrm{O}_{2}$ atmosphere. It is well known from earlier studies that $\mathrm{O}_{2}$ quenches triplet states. Using model parameters that accurately reproduce the triplet-singlet gap of $\mathrm{O}_{2}$, we have shown that the triplet states of a polyene are efficiently quenched by oxygen molecule. ${ }^{40}$ If the origin of the low energy EL peak is due to keto-defects, then one should observe this peak in oxygen atmosphere as in nitrogen atmosphere; however the peak has a large intensity in $\mathrm{N}_{2}$ atmosphere compared to the intensity in $\mathrm{O}_{2}$ atmosphere. Our theoretical studies presented here corroborate earlier experimental results and clearly show that the origin of the low energy EL peak in polyfluorene is from triplet emission. Our present time dependent studies do not incorporate the effect of electron phonon interactions and are restricted to systems of small sizes. To have a more complete understanding of the chain length dependence and the electron phonon coupling, we are investigating these nonradiative processes within the density matrix renormalization group technique. These studies will further capture other dynamical effects such as charge trapping, dissipation, and vibronic coupling.

\section{ACKNOWLEDGMENTS}

We thank Mr. Tirthankar Dutta for introducing the MSD4 scheme to our group. We thank Department of Science and Technology for supporting this work through the Grant No. SR/S2/CMP-24/2003.

${ }^{1}$ M. Gross, D. C. Müller, H. G. Nothofer, U. Scherf, D. Neher, C. Bräuchle, and K. Meerholz, Nature (London) 405, 661 (2000).

${ }^{2}$ A. W. Grice, D. D. C. Bradley, M. T. Bernius, M. Inbasekaran, W. W. Wu, and E. P. Woo, Appl. Phys. Lett. 73, 629 (1998)

${ }^{3}$ T. Virgili, D. G. Lidzey, and D. D. C. Bradley, Adv. Mater. 12, 58 (2000).

${ }^{4}$ M. Grell, W. Knoll, D. Lupo, A. Meisel, T. Miteva, D. Neher, H. G. Nothofer, U. Scherf, and A. Yasuda, Adv. Mater. 11, 671 (1999).

${ }^{5}$ K. H. Weinfurtner, H. Fujikawa, S. Tokito, and Y. Taga, Appl. Phys. Lett. 76, 2502 (2000).

${ }^{6}$ V. N. Bliznyuk, S. A. Carter, J. C. Scot, G. Klärner, R. D. Miller, and D. C. Miller, Macromolecules 32, 361 (1999).

${ }^{7}$ E. Zojer, A. Pogantsch, E. Hennebicq, D. Beljonne, and J. L. Brédas, J. Chem. Phys. 117, 6794 (2002)

${ }^{8}$ E. J. W. List, R. Guentner, P. Scanducci de Freitas, and U. Scherf, Adv. Mater. 14, 374 (2002).

${ }^{9}$ P. Chen, G. Yang, T. Liu, T. Li, M. Wang, and W. Huang, Polym. Int. 55, 473 (2006).

${ }^{10}$ A. Köhler and D. Beljonne, Adv. Funct. Mater. 14, 11 (2004).

${ }^{11}$ M. Arif, S. Mukhopadhyay, S. Ramasesha, and S. Guha, Europhys. Lett. 87, 57008 (2009).

${ }^{12}$ Y. Cao, I. D. Parker, G. Yu, C. Zhang, and A. J. Heger, Nature (London) 397, 414 (1999).

${ }^{13}$ T. M. Hong and H. F. Meng, Phys. Rev. B 63, 075206 (2001).

${ }^{14}$ M. N. Kobrak and E. R. Bittner, Phys. Rev. B 62, 11473 (2000).

${ }^{15}$ S. Karabunarliev and E. R. Bittner, J. Chem. Phys. 119, 3988 (2003).

${ }^{16}$ W. Barford, Phys. Rev. B 70, 205204 (2004).

${ }^{17}$ K. Tandon, S. Ramasesha, and S. Mazumda, Phys. Rev. B 67, 045109 (2003).

${ }^{18}$ S. Mukamel, S. Tretiak, T. Wagersreiter, and V. Chernyak, Science 277, 781 (1997).

${ }^{19}$ J. A. Pople, Trans. Faraday Soc. 49, 1375 (1953); R. Pariser and R. G. Parr, J. Chem. Phys. 21, 767 (1953).

${ }^{20}$ K. Ohno, Theor. Chim. Acta 2, 219 (1964).

${ }^{21}$ C. Schweitzer and R. Schmidt, Chem. Rev. (Washington, D.C.) 103, 1685 (2003).

${ }^{22}$ B. F. Minaev, Russ. Chem. Rev. 76, 1059 (2007)

${ }^{23}$ B. F. Minaev and H. Ågren, Int. J. Quantum Chem. 57, 519 (1996).

${ }^{24}$ H. Tsubomura and R. S. Mülliken, J. Am. Chem. Soc. 82, 5966 (1960).

${ }^{25}$ G. J. Hoijtink, Mol. Phys. 3, 67 (1960).

${ }^{26}$ Z. Barandiaran, L. Seijo, S. Huzinaga, and M. Klobukowski, Int. J. Quantum Chem. 29, 1047 (1986)

${ }^{27}$ N. V. Smith, G. K. Wertheim, S. Hüfner, and M. M. Traum, Phys. Rev. B 10, 3197 (1974).

${ }^{28}$ B. F. Minaev, V. V. Kukueva, and H. Ågren, J. Chem. Soc., Faraday Trans. 90, 1479 (1994).

${ }^{29}$ A. Askar and A. S. Cakmak, J. Chem. Phys. 68, 2794 (1978).

${ }^{30}$ T. Iitaka, Phys. Rev. E 49, 4684 (1994).

${ }^{31}$ I. D. Albert, Ph.D. thesis, Indian Institute of Sciences, Bangalore, India, 1991.

${ }^{32}$ M. Kasha, Discuss. Faraday Soc. 9, 14 (1950).

${ }^{33}$ K. Schulten, I. Ohmine, and M. Karplus, J. Chem. Phys. 64, 4422 (1976).

${ }^{34}$ D. J. Tannor, Introduction to Quantum Mechanics: A Time-Dependent Perspective (University Science Book, Sausalito, 2007).

${ }^{35}$ L. Allen and J. H. Eberly, Optical Resonance and Two-Level Atoms (Dover, New York, 1987).

${ }^{36}$ E. Merzbacher, Quantum Mechanics (Wiley, New York, 1970).

${ }^{37}$ R. W. Boyd, Nonlinear Optics (Academic, San Diego, 2003).

${ }^{38}$ C. Rothe, S. King, and A. Monkman, Nature Mater. 5, 463 (2006).

${ }^{39}$ P.-I. Lee, S. L. C. Hsu, and C. T. Chung, Synth. Met. 156, 907 (2006).

${ }^{40}$ A. J. McLean and M. A. J. Rodgers, J. Am. Chem. Soc. 115, 9874 (1993). 
${ }^{41}$ S. Ramasesha, S. Mazumdar, K. Tandon, and M. Das, Synth. Met. 139, 917 (2003).

${ }^{42}$ M. Das, S. Ramasesha, and S. Mazumdar, Synth. Met. 155, 270 (2005).

${ }^{43}$ M. Wohlgenannt, K. Tandon, S. Mazumdar, S. Ramasesha, and Z. V. Vardeny, Nature (London) 409, 494 (2001).
${ }^{44}$ F. Laquai, C. Im, A. Kadashchuk, and H. Bässler, Chem. Phys. Lett. 375, 286 (2003).

${ }^{45}$ M. Reufer, M. J. Walter, P. G. Lagoudakis, A. B. Hummel, J. S. Kolb, H. G. Roskos, U. Scherf, and J. M. Lupton, Nature Mater. 4, 340 (2005). 